\title{
Propriedades Psicométricas do Inventário de Percepção de Suporte Familiar (IPSF)
}

\section{Psychometric Properties of the Family Support Perception Inventory (IPSF)}

\section{Propiedades Psicométricas del Inventario de Percepción de Apoyo Familiar (IPSF)}

\author{
Helder Henrique Viana Batista, ORCID 0000-0001-5588-8682 \\ André Pereira Gonçalves, ORCID 0000-0002-2470-4040 \\ Ana Celi Pallini, ORCID 0000-0002-1348-2323 \\ Andréia Maria de Britto Campos, ORCID 0000-0001-5859-3265 \\ Makilim Nunes Baptista, ORCID 0000-0001-6519-254X
}

\section{Universidade São Francisco, Brasil}

\begin{abstract}
Resumo: O estudo buscou evidências de validade baseadas na estrutura interna (CFA) e na relação com variáveis externas para o Inventário de Percepção de Suporte Familiar (IPSF) e analisou o impacto da aquiescência. Participaram 234 pessoas, com idades entre 18 e 68 anos $(M=31,66$; $D P=11,75)$. Foram utilizados o IPSF e Escala Baptista de Depressão-Versão Triagem (EBADEPTriagem), a Escala de Percepção do Suporte Social-Adulto (EPSUS-A) e a Escala de Autorregulação Emocional-Adulto (EARE-AD). Foram acrescentados itens inversos ao IPSF para aquiescência. Os resultados indicaram que a estrutura bifator foi que melhor se ajustou aos dados. Além disso, a aquiescência impactou tanto nos índices de ajuste quanto nas cargas fatoriais do fator geral. As correlações do IPSF foram positivas com EPSUS-A e EARE-AD e negativas com a EBADEPTriagem. Estes resultados indicam evidências de validade para o IPSF e indica que o controle da aquiescência pode impactar escores obtidos com a aplicação da escala.
\end{abstract}

Palavras-chave: avaliação psicológica; estados emocionais; psicometria; relações familiares; autocontrole

\begin{abstract}
The study sought evidence of validity based on internal structure (CFA) and relationship to external variables for the Family Support Perception Inventory (IPSF) and analyzed the impact of acquiescence. Participants were 234 people, aged between 18 and 68 years $(\mathrm{M}=31.66$; $\mathrm{SD}=11.75)$. The IPSF, the Baptista Depression Scale - Screening Version (EBADEP-Screening), the Adult Social Support Perception Scale (EPSUS-A) and the Adult Emotional Self-Regulation Scale (EARE-AD) were used. Inverse items were added to the IPSF to check for acquiescence. The results indicated that the bifactor structure fits the data best. Also, acquiescence had an impact on both the fit indices and the factor loadings of the general factor. The IPSF correlations were positive with the EPSUS-A and EARE-AD and negative with the EBADEP-Screening. These results indicate evidence of validity for the IPSF and show that acquiescence control can impact the scores obtained when applying the scale.
\end{abstract}

Keywords: psychological assessment; emotional states; psychometrics; family relations; self-control 
Resumen: El estudio buscó evidencia de validez basada en la estructura interna (CFA) y la relación con variables externas para el Inventario de Percepción de Apoyo Familiar (IPSF) y analizó el impacto de la aquiescencia. Participaron 234 personas, con edades comprendidas entre 18 y 68 años $(M=31.66 ; D E=11.75)$. Se utilizaron IPSF y la Escala de Versión de Detección de Depresión Baptista (EBADEP-Screening), la Escala de Percepción de Apoyo Social para Adultos (EPSUS-A) y la Escala de Autorregulación Emocional de Adultos (EARE-AD). Se han agregado elementos inversos a la IPSF para aquiescencia. Los resultados indicaron que la estructura bifactor se ajusta mejor a los datos. Además, la aquiescencia impactó tanto en los índices de ajuste como en las cargas de factores del factor general. Las correlaciones de IPSF fueron positivas con EPSUS-A y EARE-AD y negativas con EBADEP-Screening. Estos resultados indican evidencia de validez para el IPSF e indican que el control de aquiescencia puede afectar los puntajes obtenidos al aplicar la escala.

Palabras clave: evaluación psicológica; estados emocionales; psicometría; relaciones familiares; autocontrol

Recebido: 10/12/2019

Aceito: $12 / 03 / 2021$

Como citar:

Viana Batista, H. H., Pereira Gonçalves, A., Celi Pallini, A., De Britto Campos, A. M. \& Nunes Baptista, M. (2021). Propriedades Psicométricas do Inventário de Percepção de Suporte Familiar (IPSF). Ciencias Psicológicas, 15(1), e-1976. doi: https://doi.org/10.22235/cp.v15i1.1976

Correspondência: Helder Henrique Viana Batista. Mestre em Psicologia, Doutorando do Programa de Pós-Graduação Stricto Senso em Psicologia da Universidade São Francisco, Brasil. E-mail: helder.hvb@gmail.com

A família é o primeiro núcleo social do ser humano, sendo a responsável pela transmissão de cuidados, valores e conhecimentos, além de suprir as necessidades iniciais da vida (Baptista, 2007; Mattanah, Lopez \& Govern, 2011). Lane (1984) destacou que o papel da família no desenvolvimento social e emocional de seus membros é fundamental, uma vez que, ela é tida como mediadora da relação indivíduo-sociedade.

As concepções de família sofreram diversas modificações ao longo do tempo, desta forma, ela pode ser entendida e abordada de diferentes perspectivas (Wagner, Tronco \& Armani, 2011). Na ciência, tais mudanças foram observadas e acompanhadas produzindo efeitos e influências na forma de estudar e trabalhar a temática (Darling \& Steinberg, 1993; Kopala-Sibley et al., 2017; Silva, Scorsolini-Comin, \& Santos, 2017; Ventura \& Noronha, 2014). À título de exemplo, McFarlane, Bellissimo e Norman (1995), consideraram importante diferenciar os conceitos "estrutura familiar" de "suporte familiar", uma vez que, a família frequentemente era destacada como favorecedora ou limitadora do desenvolvimento. Os autores ressaltaram que a estrutura familiar ou configuração familiar (entendida como a quantidade de pessoas que pertencem a uma determinada família e quem são tais pessoas, por exemplo, pai, mãe, irmãos, entre outros), pouco interferia na forma como os membros se desenvolviam. Já o suporte, atrelado às trocas afetivas e à qualidade das relações entre as pessoas que convivem como família, independente de uma estrutura específica, tinham maior impacto sobre o desenvolvimento, não obstante se o impacto era positivo ou negativo.

A presença de um suporte familiar adequado esteve atrelada a aspectos saudáveis e positivos, como melhor qualidade de vida e das relações interpessoais, saúde mental, habilidades e estratégias de enfrentamento adaptativas. Por outro lado, déficits nesse suporte e relações familiares conturbadas aumentavam significativamente o risco de depressão, tentativas de suicídio, entre outros aspectos (Lo, Kwok, Yeung, Low, \& Tam, 2017; Loton \& Waters, 2017; Magnani \& Staudt, 2018; Souza, 
Baptista \& Alves, 2008). Avaliar a qualidade do suporte torna-se fundamental para o trabalho em diversos contextos, principalmente para profissionais da psicologia, sejam em âmbito clínico, social/comunitário, saúde, trabalho ou escolar (Gonçalves, Baptista \& Farcas, 2016).

No contexto brasileiro, o Inventário de Percepção de Suporte Familiar (IPSF) foi desenvolvido por Baptista (2005) para avaliar o suporte familiar em três aspectos, quais sejam, Afetividade, Adaptação e Autonomia Familiar. A Afetividade se refere às relações afetivas positivas (carinho, proximidade, habilidade na resolução de problemas, clareza em papéis e regras dos integrantes da família). A Adaptação Familiar diz respeito aos sentimentos negativos sobre a família (raiva, isolamento, exclusão, vergonha, incompreensão e interesse). Por fim, a Autonomia Familiar se trata das relações de confiança, liberdade e privacidade (Baptista, 2009). Alguns estudos investigaram o suporte familiar por meio do IPSF, sendo identificadas associações negativas com sintomas depressivos e associações positivas com percepção de suporte social e autorregulação emocional (Baptista, Carneiro \& Sisto, 2010; Baptista \& Cremasco, 2013; Borges \& Pacheco, 2018; Cardoso \& Baptista, 2015).

Baptista et al. (2010) investigaram as associações entre o suporte familiar e a depressão em uma amostra de universitários com idades entre 18 e 52 anos. Foi identificada uma associação negativa e fraca entre os construtos $(r=-0,36)$. Em outro estudo, Baptista e Cremasco (2013) avaliaram, em crianças e adolescentes com idades entre 8 e 17 anos, as associações entre depressão, suporte familiar e suporte social. Suporte familiar apresentou correlações negativas com depressão $(r$ entre -0,23 e -0,61) e positivas com suporte social ( $r$ entre 0,30 e 0,65 ). De forma semelhante, Cardoso e Baptista (2015) identificaram associações positivas entre os suportes social e familiar $(r$ entre 0,21 e 0,45$)$ em universitários com idades entre 18 e 61 anos. Ressalta-se que no presente estudos são utilizadas versões ou escalar mais escalas diferentes das utilizadas nos estudos anteriores, além da relação com um construto ainda não explorado (autorregulação emocional), justificando explorar estas correlações.

Evidencia-se assim que o IPSF, desde sua publicação, tem apresentado índices psicométricos adequados e os estudos com sua utilização coincidem com aspectos teóricos já estabelecidos na literatura (Batista \& Noronha, 2018; Cruvinel \& Boruchovitch, 2011; Pinto, Carvalho \& Sá, 2014). Porém, apesar dos aspectos citados, não se sabe se os resultados são impactados por diferentes estilos de resposta, uma vez que, por ser um instrumento de autorrelato, as variáveis podem ser facilmente manipuladas (Wetzel \& Greiff, 2018). Os estilos de resposta são tendências do avaliado a responder a partir de um padrão, que podem ser influenciadas pelo ambiente, como desejabilidade social e respostas falsas, ou pela forma como o sujeito costuma se comportar. Como exemplo se incluem as respostas extremas, a aquiescência e a desaquiescência. Quando não controlados, esses tipos de respostas podem enviesar os resultados (Van Vaerenbergh \& Thomas, 2012). Mais especificamente, a aquiescência é um estilo de resposta no qual o respondente tende a concordar com o item, independentemente de seu conteúdo, aspecto que pode inviabilizar ou inflar os resultados do teste (Cronbach, 1942; Ferrando, Condon, \& Chico, 2004).

Desta forma, considerando que os testes precisam constantemente ter suas propriedades testadas e reformulações que propiciem melhoras na forma de medir e interpretar os dados (Borsa \& Seize, 2017; Urbina, 2009), o principal objetivo do presente estudo foi buscar evidências de validade para o IPSF com base na estrutura interna utilizando análises fatoriais confirmatórias (CFA) e com base nas relações com variáveis externas (depressão, suporte social e autorregulação emocional). Posteriormente, também foi verificado se a aquiescência interfere na estrutura interna e nas cargas fatoriais dos itens do IPSF. Diante dos objetivos expostos, hipotetiza-se que: H1) a estrutura interna encontrada corresponderá a teoria de base do instrumento (Baptista, 2005; 2007; 2009); H2) o controle de aquiescência impactará na estrutura fatorial da escala, nos índices de ajuste e nas cargas fatoriais dos itens do IPSF; H3) as correlações do IPSF com a Escala Baptista de Depressão versão triagem (EBADEP-triagem) serão todas negativas (Baptista et al., 2010; Borges \& Pacheco, 2018); H4) as correlações entre o IPSF e a Escala de Autorregulação Emocional (EARE-AD) serão positivas 
(Borges \& Pacheco, 2018; Cruvinel \& Boruchovitch, 2011); H5) as correlações entre IPSF e Escala de Suporte Social (EPSUS-A) serão positivas (Cardoso \& Baptista, 2015).

\section{Método}

\section{Participantes}

Participaram deste estudo 234 pessoas, com idades entre 18 e 68 anos $(M=31,66 ; D P=11,75)$. A maioria dos participantes era do sexo feminino $(73,9 \%)$, solteiro $(61,3 \%)$ com ensino superior (43 $\%)$ e pós-graduação $(36,2 \%)$ e com representatividade maior das regiões norte $(28,7 \%)$ e sudeste do Brasil (46 \%). Em relação a estrutura familiar, 34,9 \% relataram morar com pais e irmãos, seguidos de $20 \%$ com companheiro (a) e $17,9 \%$ com companheiro (a) e filhos.

\section{Instrumentos}

Inventário de Percepção de Suporte Familiar (IPSF; Baptista, 2009). O instrumento foi desenvolvido com base no modelo teórico proposto por Olson, Russell e Sprenkle (1983) e também em medidas desenvolvidas por outros autores (Gomide, 2003; Green, Kolevzo \& Vosler, 1985; Olson, Potner \& Lavee, 1985; Parker, Tupling \& Brown, 1979). O IPSF é um instrumento de autorrelato que visa avaliar a percepção que indivíduo possui sobre o suporte que recebe da sua família. O instrumento é composto por 42 itens, respondidos em uma escala tipo Likert de três pontos, $(0=$ quase nunca ou nunca, $1=$ às vezes, e $2=$ quase sempre ou sempre). Os itens são distribuídos em três fatores, afetivo-consistente ( 21 itens; $\alpha=0,91$ ), adaptação (13 itens; $\alpha=0,90)$ autonomia ( 8 itens; $\alpha=0,78$ ), além de um escore geral (42 itens; $\alpha=0,93$ ). "Há regras sobre diversas situações na minha família" e "Eu sinto que minha família não me compreende" são exemplos de itens. Para o presente estudo foram criados seis itens inversos aos existentes na escala, dois de cada fator, para possibilitar analisar o impacto da aquiescência no instrumento. Os pares opostos são: "As pessoas da minha família se sentem distantes umas das outras e As pessoas da minha família se sentem próximas umas das outras"; "Viver com minha família é agradável e Viver com minha família é desagradável"; "Eu sinto orgulho da minha família e Eu sinto vergonha da minha família"; "Minha família controla tudo que eu faço e Minha família me dá tanta liberdade quanto quero"; "Em minha família é proibido que eu faça as coisas que eu gosto de fazer e Em minha família é permitido que eu faça as coisas que gosto de fazer"; "Os membros da minha família expressam interesse e carinho uns com os outros e Os membros da minha família são desinteressados uns com os outros".

Escala Baptista de Depressão-Versão Triagem (EBADEP-Triagem; Baptista \& Carvalho, 2018). A EBADEP-triagem foi desenvolvida com base na versão adulta da EBADEP (EBADEP-A; Baptista, 2012) e tem como objetivo avaliar os sintomas da depressão. Na EBADEP-triagem, foram selecionados 15 itens com base nos principais sintomas dos manuais psiquiátricos, para compor a escala. No estudo de Baptista e Carvalho (2018) a EBADEP-triagem foi capaz de discriminar 40 pacientes diagnosticados com depressão pela SCID-I de 40 pessoas sem depressão, com sensibilidade igual a $95 \%$ e especificidade de $87 \%$. "Não tenho vontade de chorar" e "Não consigo concentrar em minhas atividades" são exemplos de itens. Na amostra do presente estudo, a fidedignidade por consistência interna da EBADEP-triagem foi igual a 0,78. A consistência interna da escala com amostra deste estudo foi $\alpha=.93$.

Escala de Percepção do Suporte Social-Adulto (EPSUS-A; Cardoso \& Baptista, 2016). Criada com base na teoria de Rodriguez e Cohen (1998), a escala avalia a percepção do respondente em relação ao suporte social recebido. É composta por 36 itens com escala tipo Likert quatro pontos $(0=$ nunca e $4=$ sempre). Os itens se agrupam em quatro fatores, a saber, afetivo (17 itens; $\alpha=0,92)$, interações sociais ( 5 itens; $\alpha=0,75)$, instrumental ( 7 itens; $\alpha=0,82$ ) e enfrentamento de problemas ( 7 itens; $\alpha=0,83)$. "Me fornecem alimentação quando preciso" e "São agradáveis para se conversar" são exemplos de itens. 
Escala de Autorregulação Emocional-Adulto (EARE-AD; Noronha, Baptista \& Batista, 2019). A teoria que embasou a construção da EARE-AD foi a de Gratz e Roemer (2004), além de contribuições de Thompson (1994), Berking, Ebert, Cuijpers, \& Hofmann, (2013) e Weiss, Gratz e Lavender (2015). A escala avalia a capacidade de controle das emoções frente a situações que geram tristeza. É composta por 34 itens, respondidos em uma escala Likert de cinco pontos $(0=$ nunca $\mathrm{e} 4=$ sempre) e quanto maior a pontuação maior a autorregulação emocional. Os itens são agrupados em 4 fatores, a saber, estratégias de enfrentamento adequadas ( 15 itens; $\alpha=0,98$ ), pessimismo ( 6 itens; $\alpha=$ 0,88), paralisação (6 itens; $\alpha=0,92$ ) e externalização de agressividade (7 itens; $\alpha=0,69$ ). As pontuações dos fatores 2, 3 e 4 devem ser invertidas. O respondente precisa considerar a frase "Quando estou triste..." para responder os itens. "Tento pensar em outras coisas" e "Maltrato as pessoas" são exemplos de itens.

\section{Procedimento}

Este estudo seguiu as normas éticas de pesquisas com seres humanos e foi aprovado pelo Comitê de Ética em Pesquisa da Universidade de São Francisco. A coleta de dados foi realizada por meio de um link do Google Forms e os participantes receberam convites para participação na pesquisa nas redes sociais (WhatsApp, Facebook). Ao concordar em participar da pesquisa por meio do Termo de Consentimento Livre e Esclarecido, os participantes responderam o questionário sociodemográfico, EBADEP-Triagem, IPSF, EPSUS e EARE-AD.

\section{Análise de dados}

Os programas utilizados na análise de dados foram o SPSS 20.1 e Mplus 7.11. Foi criado um índice de aquiescência somando as respostas nos pares de itens opostos e calculando as médias que posteriormente foram utilizadas no Multiple Indicator Multiple Cause (MIMIC; Muthén, 1989) para verificar o impacto da aquiescência sobre os itens da escala. Com intuito de verificar a estrutura interna da escala e o impacto da aquiescência, foram testados dois modelos de análise fatorial confirmatória (CFA) para o IPSF partindo da teoria de construção desta escala (Baptista, 2005): três fatores de primeira ordem (CFA-1); três fatores de primeira ordem com controle de aquiescência (CFA-2). Além disso, com objetivo de verificar a existência de uma mesma variável latente explicando os itens e, consequentemente, uma evidência empírica para utilização do escore geral desta escala, foram testados dois modelos confirmatórios bifactor: um fator geral e três fatores específicos (CFA-3); um fator geral e três fatores específicos controlando o índice de aquiescência (CFA-4).

Foram utilizados os indicadores chi-square y degrees of freedom ratio $\left(X^{2} / d f<2\right)$, Confirmatory Fit Index (CFI; > 0.90), Tucker-Lewis Index (TLI > 0.90), e Root Mean Square Error of Approximation (RMSEA; <0.05) para verificar qual modelo melhor se adequava aos dados (Hu \& Bentler, 1999). O teste de correlação de Pearson, com significância de $p<0.05$, foi utilizado para identificar as associações entre os instrumentos, com base na interpretação de magnitudes de Levin e Fox (2004), sendo fracas $(<0.30)$, moderadas (entre 0.30 e 0,59$)$, fortes (entre 0.60 e 0.99 ) ou perfeitas (1.0). 


\section{Resultados}

As primeiras análises realizadas foram as CFA's, com base na construção do IPSF (Baptista, 2005). Os índices obtidos em cada modelo estão dispostos na Tabela 1.

Tabela 1.

Índices de ajuste e cargas fatoriais do modelo do IPSF (Baptista, 2005) testado nas análises fatoriais confirmatórias

\begin{tabular}{ccccc}
\hline Modelo & $x^{2} / \mathrm{df}$ & CFI & RMSEA & TLI \\
\hline Valor de referência & $<2$ & $\geq .90$ & $<0.05$ & $\geq .90$ \\
\hline CFA-1 & $1334.549 / 816(1.63)$ & 0.958 & 0.05 & 0.955 \\
CFA-2 & $1174.074 / 738(1.59)$ & 0.928 & 0.05 & 0.920 \\
CFA-3 & $1068.378 / 777(1.37)$ & 0.972 & 0.04 & 0.969 \\
CFA-4 & $1050.225 / 777(1.35)$ & 0.954 & 0.04 & 0.947 \\
\hline
\end{tabular}

Nota: CFA-1: três fatores de primeira ordem; CFA-2: três fatores de primeira ordem com controle de aquiescência; CFA-3: um fator geral e três específicos; CFA-4: um fator geral e três fatores específicos com controle de aquiescência; F1: afetivo-consistente; F2: adaptação; F3: autonomia; F4: fator geral.

O modelo CFA-1, com três fatores, apresentou índices de ajustes satisfatórios e as cargas fatoriais variaram entre 0.15 e 0.92 no fator afetivo consistente (apenas um item abaixo de 0.30 ); 0.56 a 0.92 no fator adaptação; e 0.60 a 0.95 no fator autonomia. Com controle de aquiescência o ajuste do modelo CFA-2 com três fatores específicos também apresentou índices de ajustes satisfatórios e as cargas fatoriais foram, de modo geral, menores quando comparado ao modelo sem controle de aquiescência, no fator afetivo consistente as cargas estiveram entre 0.19 a 0.62 (apenas um item abaixo de 0.30 ), adaptação 0.35 a 0.79 e autonomia 0.46 a 0.77 ).

O modelo com um fator geral e três específicos, CFA-3, apresentou índices de ajustes melhores quando comparado ao modelo com três fatores de primeira ordem. As cargas fatoriais variaram entre 0.23 a 0.93 no fator geral, com apenas um item com carga menor que 0.30 . Nos fatores específicos as cargas fatoriais estiveram entre 0.07 a 0.70 no afetivo-consistente, 0.27 a 0.56 no adaptação e 0.25 a 0.66 no fator autonomia. O modelo CFA-4 de fator geral com controle de aquiescência também apresentou índices de ajustes adequados. Os itens apresentaram menores cargas no fator geral após o controle da aquiescência, porém não houve alteração importantes de cargas nos fatores específicos, no fator geral as cargas foram entre 0.14 e 0.76 , (dois itens apresentaram cargas menores do que 0.30), afetivo-consistente entre 0.07 e 0.60 , adaptação entre 0.27 a 0.50 e autonomia entre 0.25 e 0.65 . A consistência interna deste modelo foi de $\alpha=0.95$ para o fator geral, $\alpha=0.88$ no afetivo-consistente, $\alpha=0.87$ na adaptação e $\alpha=0.86$ para autonomia.

Por meio do teste de correlação de Pearson foi possível identificar correlações significativas do IPSF com as variáveis externas. O IPSF total apresentou as correlações mais elevadas com as outras medidas em comparação com os três fatores do IPSF. Cabe destacar que as pontuações dos fatores pessimismo, paralisação e externalização de agressividade foram invertidos. Na Tabela 2 são apresentadas as correlações do IPSF com as variáveis externas. 
Tabela 2.

Correlações do IPSF com EARE-AD, EBADEP-Triagem e EPSUS-A

\begin{tabular}{cccccccccc}
\hline & 1 & 2 & 3 & 4 & 5 & 6 & 7 & 8 & 9 \\
\hline Afetivo- & $-0.55^{* *}$ & $0.41^{* *}$ & $0.36^{* *}$ & $0.52^{* *}$ & $0.54^{* *}$ & $0.50^{* *}$ & $0.48^{* *}$ & $0.30^{* *}$ & $0.53^{* *}$ \\
Consistente & & & & & & \\
Adaptação & $-0.53^{* *}$ & $0.33^{* *}$ & $0.36^{* *}$ & $0.46^{* *}$ & $0.47^{* *}$ & $0.47^{* *}$ & $0.42^{* *}$ & $0.30^{* *}$ & $0.46^{* *}$ \\
Autonomia & $-0.43^{* *}$ & $0.34^{* *}$ & $0.20^{* *}$ & $0.34^{* *}$ & $0.39^{* *}$ & $0.40^{* *}$ & $0.34^{* *}$ & $0.19^{* *}$ & $0.38^{* *}$ \\
IPSF Total & $-0.59^{* *}$ & $0.42^{* *}$ & $0.38^{* *}$ & $0.53^{* *}$ & $0.56^{* *}$ & $0.53^{* *}$ & $0.49^{* *}$ & $0.32^{* *}$ & $0.55^{* *}$ \\
\hline
\end{tabular}

Nota: $*=\mathrm{p}<0.05 ; * *=\mathrm{p}<0.01 ; 1=$ EBADEP-Triagem; $2=-$ Estratégias de enfretamento adequadas (EARE-AD); $3=$ Externalização de agressividade (EARE-AD); 4 = Pessimismo (EARE-AD); 5 = Paralisação (EARE-AD); 6= Afetivo (EPSUS-A); $7=$ Interações Sociais (EPSUS-A); $8=$ Instrumental (EPSUS-A); 9 = Enfrentamento de Problemas (EPSUSA).

As associações entre suporte familiar e depressão foram todas negativas, com magnitudes moderadas. As correlações entre o IPSF e as medidas de suporte social e autorregulação emocional foram todas positivas, com magnitudes variando entre fracas e moderadas.

\section{Discussão}

O objetivo do presente estudo foi buscar evidências de validade com base na estrutura interna e nas relações com variáveis externas (depressão, suporte social e autorregulação emocional) para o IPSF. Além de verificar se a aquiescência interfere nos resultados do IPSF. As hipóteses elaboradas para este estudo foram, de modo geral corroboradas. H1) os três fatores de primeira ordem foram recuperados conforme esperado, porém, os resultados indicaram a possibilidade de existência de um fator geral. $\mathrm{O}$ controle da aquiescência impactou na estrutura fatorial do instrumento, nos índices de ajustes e nas cargas fatoriais dos itens no fator geral (H2). As correlações do IPSF com a EBADEPtriagem foram negativas (H3); correlações entre o IPSF e a escala de EARE-AD foram positivas (H4) e por fim, as correlações entre IPSF e EPSUS-A também foram positivas (H5).

A estrutura interna do IPSF esteve em consonância com literatura prévia que indicava três fatores (Baptista, 2005; 2007; 2009). Entretanto, no presente estudo um fator geral foi encontrado, o que sugere que, além dos fatores específicos previamente encontrados, há a contribuição dos itens para uma variável latente em comum. Este resultado possibilita verificar empiricamente que além da contribuição dos itens para calcular os escores para cada fator específico, um escore geral de suporte familiar pode ser obtido. O modelo bifactor, porém, pode estar relacionado com problemas de método, sendo esse um dos problemas da aquiescência. A aquiescência refere-se à tendência de responder positivamente aos itens, sem considerar o conteúdo descrito, distanciando os escores obtidos com aplicação do instrumento do escore verdadeiro do sujeito (Billiet \& McClendon, 2000). Os resultados obtidos com o controle de aquiescência, diminuíram as cargas do fator geral, o que demonstra que a aquiescência pode estar afetando os escores oriundos da aplicação, e que o controle deste viés no IPSF seria importante para este instrumento. Ressalta-se que apesar do impacto da aquiescência no fator geral, quando esta é controlada, mesmo com cargas menores, o fator geral se mantém e o modelo bifactor ainda é o modelo que melhor se ajusta aos dados. Apesar disso, os dois itens com cargas menores que 0,30 no fator geral demostra não estar funcionando com o controle de aquiescência, o que pode gerar exclusão destes itens no instrumento.

$\mathrm{Na}$ hipótese 3 era esperado que as associações entre o IPSF e a EBADEP-Triagem fossem negativas. Os resultados encontrados nas correlações entre o IPSF e a EBADEP-Triagem são coerentes e similares à literatura (Baptista et al., 2010; Baptista, Souza \& Alves, 2008; Borges \& Pacheco, 2018), confirmando a hipótese 3 do presente estudo. O suporte familiar envolve a percepção de carinho, de relações colaborativas e compreensão entre os membros da família, habilidades de estratégia de enfrentamento, autonomia e confiança (Baptista, 2005; Baptista et al., 2010). Assim, os resultados indicam que a família e o suporte que ela pode proporcionar estão associados à saúde 
mental das pessoas podendo ser considerado um fator de proteção contra o desenvolvimento de sintomatologia depressiva (Baptista et al., 2008; Borges \& Pacheco, 2018).

Ademais, há de se considerar que as correlações entre os fatores total e afetivo-consistente do IPSF com a depressão tiveram magnitudes mais elevadas quando comparadas a amostras de crianças e adolescentes, ao passo que com o fator adaptação as correlações foram menores (Borges \& Pacheco, 2018; Cruvinel \& Boruchovitch, 2011). Aventa-se a possibilidade de a faixa etária ser uma variável que pode impactar na percepção de suporte familiar, remetendo a possibilidade do desenvolvimento de versões específicas para ciclos desenvolvimentais diferentes e/ou normas específicas (Baptista \& Cremasco, 2013).

Em relação à hipótese 4, esperava-se que as associações entre os fatores do IPSF e da EAREAD fossem positivas após a inversão dos fatores pessimismo, externalização de agressividade e paralisação (EARE-AD). Os resultados confirmaram a hipótese e são coerentes com os dados identificados em outros estudos (Borges \& Pacheco, 2018; Cruvinel \& Boruchovitch, 2011; Gratz \& Roemer, 2004). A justificativa para os resultados pode ser o fato da autorregulação emocional, quando em baixos níveis, estar associada às dificuldades no estabelecimento de relacionamentos interpessoais saudáveis e acarretar em problemas emocionais (Gratz \& Roemer, 2004; Simon \& Durand-Bush, 2015). A dificuldade de alguns indivíduos em relação à administração das próprias emoções poderia impactar negativamente no bem-estar e na convivência familiar (Tamir, 2015), não percebendo o suporte que os familiares oferecem. Nesse sentido, faz-se importante considerar o funcionamento familiar e as características pessoais de cada indivíduo para minimizar interpretações isoladas, por exemplo, aquelas que focam somente nos pais ou somente nos filhos (Noronha \& Batista, 2017; Weber, Bradenburg \& Viezzer, 2003).

A hipótese 5 também foi confirmada no presente estudo. Esperava-se que as associações entre o IPSF e a EPSUS-A fossem positivas. Como identificado em estudos anteriores (Baptista \& Cremasco, 2013; Cardoso \& Baptista, 2015), as associações entre os suportes familiar e social foram positivas. Tais resultados podem ser explicados pelo fato do suporte familiar ser uma espécie de dimensão reduzida do suporte social (Baptista, Cardoso \& Gomes, 2012; Duru, 2007). Quando comparadas ao estudo de Cardoso e Baptista (2015), as magnitudes encontradas na presente investigação foram mais altas. Ademais, tanto no presente estudo quanto no de Cardoso e Baptista (2015) as associações entre os fatores do IPSF e o fator Instrumental da EPSUS-A foram as que apresentaram magnitudes menores. $\mathrm{O}$ fator Instrumental diz respeito a percepção do indivíduo tem no que se refere às ajudas financeiras e práticas que recebe dos membros de sua família (Baptista et al., 2012). Esses resultados parecem indicar que as questões que permeiam o desenvolvimento familiar vão além de aspectos materiais, sendo necessário levar em conta os aspectos emocionais que circundam as relações entre membros de uma família (Baptista et al., 2012; Noronha \& Batista, 2017).

Além disso, há de se considerar as características da amostra e seus possíveis impactos nos dados do IPSF. À título de exemplo, apenas $34 \%$ da amostra residia com pais e irmãos. Ou seja, é possível que a maior parte dos participantes tenha saído de casa por ter desenvolvido certa independência e autonomia da família, sobretudo ao se considerar que se tratam de adultos jovens (Carter \& McGoldrick, 1995; Pellegrini, Silva, Barreto \& Crepaldi, 2015). Dessa forma, os dados precisam ser analisados com cautela, uma vez que a fase da adultez jovem é marcada por uma diferenciação do self em relação aos familiares, permeando noções de hierarquia entre pais e filhos, e a escolha por uma profissão e relacionamentos íntimos (Carter \& McGoldrick, 1995; Fiorini, Moré, \& Bardagi, 2017). Pode ser que, em alguns casos, o suporte familiar tenha sido de uma forma, porém, por ser um período de transição permeado de estresse e ansiedade (Aylmer, 1995), tal percepção tenha se alterado.

Os resultados obtidos no presente estudo demonstraram consistentes evidências de validade para o IPSF com base na estrutura interna e na relação com variáveis externas. Os achados parecem indicar que o IPSF pode ser impactado pelo estilo de resposta aquiescente, o que permite levantar a 
hipótese de que controlando esse viés, os escores obtidos podem ser mais próximos do escore verdadeiro do sujeito. Algumas limitações deste estudo precisam ser ponderadas: (a) o número da amostra pode ter impactado nos resultados obtidos; (b) a escala não foi totalmente balanceada para o controle de aquiescência, ou seja, não foram criados pares opostos para todos os itens e sim dois para cada fator; (c) as características sociodemográficas dos participantes são pouco representativas da população brasileira (predominantemente mulheres, solteiros e com ensino superior completo). Sugere-se que estudos futuros verifiquem o impacto da aquiescência com a escala totalmente balanceada e, além disso, possa controlar também a desejabilidade social dos itens e identificar possíveis interferências das variáveis de caracterização dos participantes.

\section{Financiamento}

O presente trabalho foi realizado com apoio da Coordenação de Aperfeiçoamento de Pessoal e Nível Superior - Brasil (Capes) - Código de Financiamento 001.

\section{Referências}

Aylmer, R. C. (1995). O lançamento do jovem adulto solteiro. Em B. Carter \& M. McGoldrick, (1995). As mudanças no ciclo de vida familiar: uma estrutura para a terapia familiar (pp. 169-183). (M. A. V. Veronese, Trad., 2ed ${ }^{\text {ed }}$. Porto Alegre: Artes Médicas.

Baptista, M. N., \& Carvalho, L. F. (2018). Diagnostic accuracy of a Brazilian depression self-report measure (EBADEP): Original and short versions. Avaliação Psicológica, 17(4), 484-492. doi: 10.15689/ap.2018.1704.8.08

Baptista, M. N., \& Cremasco, G. S. (2013). Propriedades psicométricas da Escala Baptista de Depressão Infanto-juvenil (EBADEP-IJ). Arquivos Brasileiros de Psicologia, 65(2), 198-213.

Baptista, M. N., Cardoso, H. F., \& Gomes, J. O. (2012). Intergeracionalidade familiar. Em M. N. Baptista, \& M. L. M. Teodoro (Orgs.), Psicologia de família: teoria, avaliação e intervenção (pp. 16-26). Porto Alegre: Artmed.

Baptista, M. N., Carneiro, A. M., \& Sisto, F. F. (2010). Estudo psicométrico de Escalas de Depressão (EDEP e BDI) e o Inventário de Percepção de Suporte Familiar (IPSF). Psicologia em Pesquisa, 4(1), 65-73. doi: 10.24879/201000400100345

Baptista, M. N., Souza, M. S., \& Alves, G. A. S. (2008). Evidencias de validade entre a Escala de Depressão-EDEP, o BDI e o Inventário de Percepção de Suporte Familiar - IPSF. Psico-USF, 13(1), 211-220. doi: 10.1590/S1413-82712008000200008

Baptista. M. N. (2005). Desenvolvimento do Inventário de Percepção de Suporte Familiar (IPSF): estudos psicométricos preliminares. Psico-USF, 10(1), 11-19. doi: 10.1590/S141382712005000100003

Baptista. M. N. (2007). Inventário de percepção de suporte familiar (IPSF): estudo componencial em duas configurações. Psicologia: Ciência e Profissão, 27(3), 496-509. doi: 10.1590/S141498932007000300010

Baptista. M. N. (2009). Inventário de Percepção do Suporte Familiar (IPSF). Vetor: São Paulo.

Baptista. M. N. (2012). Manual técnico da Escala Baptista de Depressão em Adultos (EBADEP-A). São Paulo: Vetor.

Batista, H. H. V. \& Noronha, A. P. P. (2018). Instrumentos de autorregulação emocional: uma revisão de literatura. Avaliação Psicológica, 17(3), 389-398. doi: 10.15689/ap.2018.1703.15643.12

Berking, M., Ebert, D., Cuijpers, P., \& Hofmann, S. G. (2013). Emotion-regulation skills training enhances the efficacy of cognitive behavioral therapy for major depressive disorder. Psychotherapy and Psychosomatics, 82(4), 234-245. doi: 10.1159/000348448 
Billiet, J. B., \& McClendon, M. J. (2000). Modeling acquiescence in measurement models for two balanced sets of items. Structural Equation Modeling, 7(4), 608-628. doi: 10.1207/S15328007SEM0704_5

Borges, L., \& Pacheco, J. T. B. (2018). Sintomas depressivos, autorregulação emocional e suporte familiar: Um estudo com crianças e adolescentes. Estudos Interdisciplinares em Psicologia, 9(3), 132-148. doi: 10.5433/2236-6407.2018v9n3suplp132

Borsa, J. C., \& Seize, M. M. (2017). Construção e adaptação de instrumentos psicológicos: Dois caminhos possíveis. Em B. F. Damásio, \& J. C. Borsa (Orgs), Manual de desenvolvimento de instrumentos psicológicos (pp. 15-38). São Paulo: Vetor.

Cardoso, H.F., Baptista, M.N. (2016). EPSUS-A: Escala de Percepção de Suporte Social (versão adulto). São Paulo: CETEPP-Hogrefe.

Cardoso. H. F. \& Baptista. M. N. (2015). Evidência de validade para a Escala de Percepção do Suporte Social (Versão Adulta) - EPSUS-A: um estudo correlacional. Psicologia: Ciência e Profissão, 35(3), 946-958. doi: 10.1590/1982-3703001352013

Carter, B., \& McGoldrick, M. (1995). As mudanças no ciclo de vida familiar: uma estrutura para a terapia familiar (M. A. V. Veronese, Trad.). 2ed. Porto Alegre: Artes Médicas.

Cronbach, L. J. (1942). Studies of acquiescence as a factor in the true-false test. Journal of Educational Psychology, 33(6), 401-415.

Cruvinel, M., \& Boruchovitch, E. (2011). Regulação emocional em crianças com e sem sintomas de depressão. Estudos de Psicologia (Natal), 16(3), 219-226. doi: 10.1590/S1413294X2011000300003

Darling, N., \& Steinberg, L. (1993). Parenting style as context: An integrative model. Psychological Bulletin, 113(3), 487-496. doi: 10.1037/0033-2909.113.3.487

Duru, E. (2007). Re-examination of the psychometric characteristics of the multidimensional scale of perceived social support among Turkish University students. Social Behavior and Personality, 35(4), 443-452. doi: 10.2224/sbp.2007.35.4.443

Ferrando, P. J., Condon, L., \& Chico, E. (2004). The convergent validity of acquiescence: An empirical study relating balanced scales and separate acquiescence scales. Personality and Individual Differences, 37(7), 1331-1340. doi: 10.1016/j.paid.2004.01.003

Fiorini, M. C., Moré, C. L. O. O., \& Bardagi, M. P. (2017). Família e desenvolvimento de carreira de jovens adultos no context brasileiro: revisão integrativa. Revista Brasileira de Orientação Profissional, 18(1), 43-55. doi: 10.26707/1984-7270/2017v18n1p43

Gomide, P. I. C. (2003). Estilos parentais e comportamento antissocial. Em A. Del Prette \& Z. Del Prette (Eds.). Habilidades sociais, desenvolvimento e aprendizagem: questões conceituais, avaliação e intervenção (pp. 21-60). Campinas: Alínea.

Gonçalves, M., Baptista, M. N., \& Farcas, D. (2016). IPSF: análise da estrutura interna em uma amostra de jovens portugueses adultos. Avaliação Psicológica, 15(1), 115-123. doi: 10.15689/ap.2016.1501.12

Gratz, K. L., \& Roemer, L. (2004). Multidimensional assessment of emotion regulation and dysregulation: Development factor structure, and initial validation of the Dificulties in Emotion Regulation Scale. Journal of Psychopatology and Behavioral Assessment, 26(1), 4154. doi: 10.1007/s 10862-008-9102-4

Green, R. G., Kolevzon, M. S., \& Vosler, N. R. (1985). The beavers timberlawn model of family competence and the circumplex model of family adaptability and cohesion: Separate but equal? Family Process, 24(3), 385-398. doi: 10.1111/j.1545-5300.1985.00385.x

Hu, L., \& Bentler, P. M. (1999). Cutoff criteria for fit indexes in covariance structure analysis: Conventional criteria versus new alternatives. Structural Equation Modeling: A Multidisciplinary Journal, 6(1), 1-55. doi: 10.1080/10705519909540118 
Kopala-Sibley, D. C., Jelinek, C., Kessel, E., Frost, A., Allmann, A. E. E., \& Klein, D. N. (2017). Parental depressive history, parenting styles, and child psychopathology over six years: The contribution of each parent's depressive history to the other's parenting styles. Development and Psychopathology, 29(4), 1468-1482. doi: 10.1017/S0954579417000396

Lane, S. T. M. (1984). A Psicologia Social e uma nova concepção de homem para a Psicologia. Em S. T. M. Lane \& W. Codo (Eds.), Psicologia Social: O homem em movimento (pp. 10-19). São Paulo: Brasiliense.

Levin, J., \& Fox, J. A. (2004). Estatística para ciências humanas. São Paulo: Pearson.

Lo, H. H. M., Kwok, S. Y. C. L., Yeung, J. W. K., Low, A. Y. T., \& Tam, C. H. L. (2017). The moderating effects of gratitude on the association between perceived parenting styles and suicidal ideation. Journal of Child and Family Studies, 26(6), 1671-1680. doi: 10.1007/s10826-017-0683-y

Loton, D. J., \& Waters, L. E. (2017). The mediating effect of self-efficacy in the connections between strength-based parenting, happiness and psychological distress in teens. Frontiers in Psychology, 8, 1-13. doi: 10.3389/fpsyg.2017.01707

Magnani, R. M., \& Staudt, A. C. P. (2018). Estilos parentais e suicidio na adolescencia: Uma reflexão acerca dos fatores de proteção. Pensando Famílias, 22(1), 75-86.

Mattanah. J. F., Lopez. F. G., \& Govern. J. M. (2011). The contributions of parental attachment bonds to college student development and adjustment: a meta-analytic review. Journal of Counseling Psychology, 58(4), 565-596. doi: 10.1037/a0024635

McFarlane, A. H., Bellissimo, A., \& Norman, G. R. (1995). The role of family and peers in social self-efficacy: Links to depression in adolescence. American Journal of Orthopsychiatry, 65(3), 402-410. doi: 10.1037/h0079655

Muthén, B. O. (1989). Tobit factor analysis. British Journal of Mathematical and Statistical Psychology, 42(2), 241-250. doi: 10.1111/j.2044-8317.1989.tb00913.x

Noronha, A. P. P. \& Batista, H. H. V. (2017). Escala de forças e estilos parentais: Estudo correlacional. Estudos Interdisciplinares em Psicologia, 8(2), 02-19. doi: 0.5433/22366407.2016v8n2p02

Noronha, A. P. P., Baptista, M. N., \& Batista, H. H. V. (2019). Estudos psicométricos iniciais da escala de autorregulação emocional: Versões adulto e infanto-juvenil. Estudos de Psicologia (Campinas), 36(e180109), 1-12. doi: 10.1590/1982-0275201936e18010

Olson, D. H., Portner, J., \& Lavee, Y. (1985). Family adaptability \& cohesion scales - FACES III (research instrument and manual). St. Paul: University of Minnesota, Family Social Science.

Olson, D. H., Russell, C. S., \& Sprenkle, D. H. (1983). Circumplex model of marital and family systems: Theoretical update. Family Process, 22(1), 69-83, doi: 10.1111/j.15455300.1983.00069.x

Parker, G., Tupling, H., \& Brown, L. B. A (1979). Parental Bonding Instrument. British Journal of Medical Psychology, 52(1), 1-10. doi:10.1111/j.2044-8341.1979.tb02487.x

Pellegrini, P. G., Silva, I. M., Barreto, M., \& Crepaldi, M. A. (2015). Diferenciação do adulto jovem: Um estudo de caso em atendimento familiar. Pensando Famílias, 19(1), 114-129.

Pinto, H. M., Carvalho, A. R., \& Sá, E. N. (2014). Os estilos educativos parentais e a regulação emocional: Estratégias de regulação e elaboração emocional das crianças em idade escolar. Análise Psicológica, 32(4), 387-400. doi: 1014417/ap.32.3.844

Rodriguez, M. S. \& Cohen, S. (1998). Social support. Encyclopedia of Mental Health, 3, 535-544.

Silva, L. A., Scorsolini-Comin, F., \& Santos, M. A. (2017). Casamentos de longa duração: recurssos pessoais como estratégias de manutenção do laço conjugal. Psico-USF, 22(2), 323-335. doi: $10.1590 / 1413-82712017220211$

Simon, C. R., \& Durand-Bush, N. (2015). Does self-regulation capacity predict psychological wellbeing in physicians? Psychology, Health and Medicine, 20(3), 311-321. doi: $10.1080 / 13548506.2014 .936887$ 
Souza. M. S., Baptista. M. N. \& Alves. G. A. S. (2008). Suporte familiar e saúde mental: evidência de validade baseada na relação entre variáveis. Aletheia. 28. 32-44.

Tamir, M. (2015). Why do people regulate their emotions? A taxonomy of motives in emotion regulation. Personality and Social Psychology Review, 20(3), 199-222. doi: $10.1177 / 1088868315586325$

Thompson, R. A. (1994). Emotion regulation: A theme in search of definition. Monographs of the Society for Research in Child Development, 59(2-3), 25-52. doi: 10.1111/j.15405834.1994.tb01276.x

Urbina, S. (2009). Fundamentos da testagem psicológica. Artmed Editora.

Van Vaerenbergh, Y., \& Thomas, T. D. (2012). Response styles in survey research: A literature review of antecedents, consequences, and remedies. International Journal of Public Opinion Research, 25(2), 195-217.doi: 10.1093/ijpor/eds021

Ventura. C. D., \& Noronha, A. P. P. (2014). Autoeficácia para escolha profissional. suporte familiar e estilos parentais em adolescentes. Avaliação Psicológica, 13(3), 317-324.

Wagner, A., Tronco, C., \& Armani, A. B. (2011). Os desafios da família contemporânea. Desafios psicossociais da família contemporânea: Pesquisas e reflexões. Porto Alegre: Artmed.

Weber, L. N. D., Brandenburg, O. J., \& Viezzer, A. P. (2003). A relação entre o estilo parental e o otimismo da criança. Psico-USF, 8(1), 71-79. doi: 10.1590/S1413-82712003000100010

Weiss, N. H., Gratz, K. L., \& Lavender, J. M. (2015). Factor structure and initial validation of a multidimensional measure of difficulties in the regulation of positive emotions: The DERSPositive. Behavior Modification, 39(3), 431-453. doi: 10.1177/0145445514566504

Wetzel, E., \& Greiff, S. (2018). The world beyond rating scales: Why we should think more carefully about the response Format in Questionnaires. European Journal of Psychological Assessment, 34(1), 1-5. doi: 10.1027/1015-5759/a000469

Participação dos autores: Participação dos autores: a) Planejamento e concepção do trabalho; b) Coleta de dados; c) Análise e interpretação de dados; d) Redação do manuscrito; e) Revisão crítica do manuscrito.

H. H. V. B. contribuiu em a, b, c, d, e; A. P. G. contribuiu em a, b, c, d, e; A. C. P. contribuiu em a, b, c, d, e; A. M. B. C. contribuiu em a, b, c, d, e; M. N. B. contribuiu em a, b, c, d, e.

Editora científica responsável: Dra. Cecilia Cracco. 